\title{
Erratum: More realistic Hamiltonians for the fractional quantum Hall regime in GaAs and graphene [Phys. Rev. B 87, 245129 (2013)]
}

Michael R. Peterson and Chetan Nayak

(Received 30 September 2015; published 19 October 2015)

In the original paper, a typographical error was introduced into the value of the $M=6$ three-body pseudopotential for the $N=1$ Landau level, which impacted two figures and one table-both are corrected below in Figs. 1 and 2 and Table I. The original figures and table that are corrected below are Figs. 9 and 11 (middle panel) and Table III (column three) in the original paper.

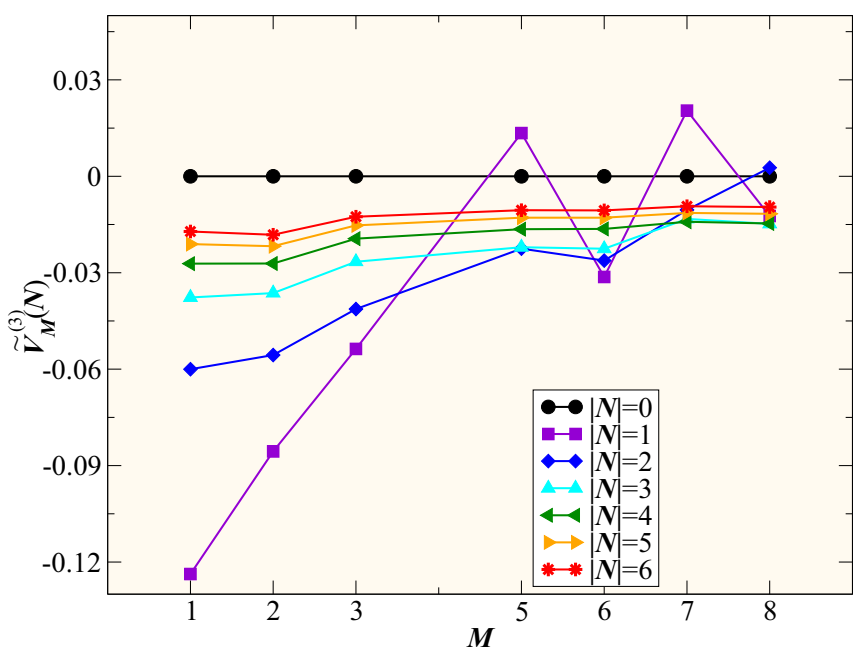

FIG. 1. (Color online) $\tilde{V}_{M}^{(3)}(N)$ as a function of $M$ for $N=0-6$. Note that for $N=0$ all three-body pseudopotentials exactly vanish. All energies are given in units of $e^{2} / \epsilon \ell_{0}$. This figure replaces Fig. 9 in the original paper.

TABLE I. We list the numerical values of the three-body pseudopotentials $\tilde{V}_{M}^{(3)}(N=1)$ as a function of $M$ for graphene. All energies are given in units of $e^{2} / \epsilon \ell_{0}$. The $M=6$ pseudopotential in bold was originally incorrectly given as positive. This table replaces the third column of Table III in the original paper.

\begin{tabular}{lr}
\hline \hline$N=$ & \multicolumn{1}{c}{1} \\
\hline$\tilde{V}_{1}^{(3)}(N)$ & -0.1237 \\
$\tilde{V}_{2}^{(3)}(N)$ & -0.0856 \\
$\tilde{V}_{3}^{(3)}(N)$ & -0.0537 \\
$\tilde{V}_{5}^{(3)}(N)$ & 0.0135 \\
$\tilde{V}_{6}^{(3)}(N)$ & $\mathbf{0 . 0 3 1 3}$ \\
$\tilde{V}_{7}^{(3)}(N)$ & 0.0205 \\
$\tilde{V}_{8}^{(3)}(N)$ & -0.0123 \\
\hline
\end{tabular}

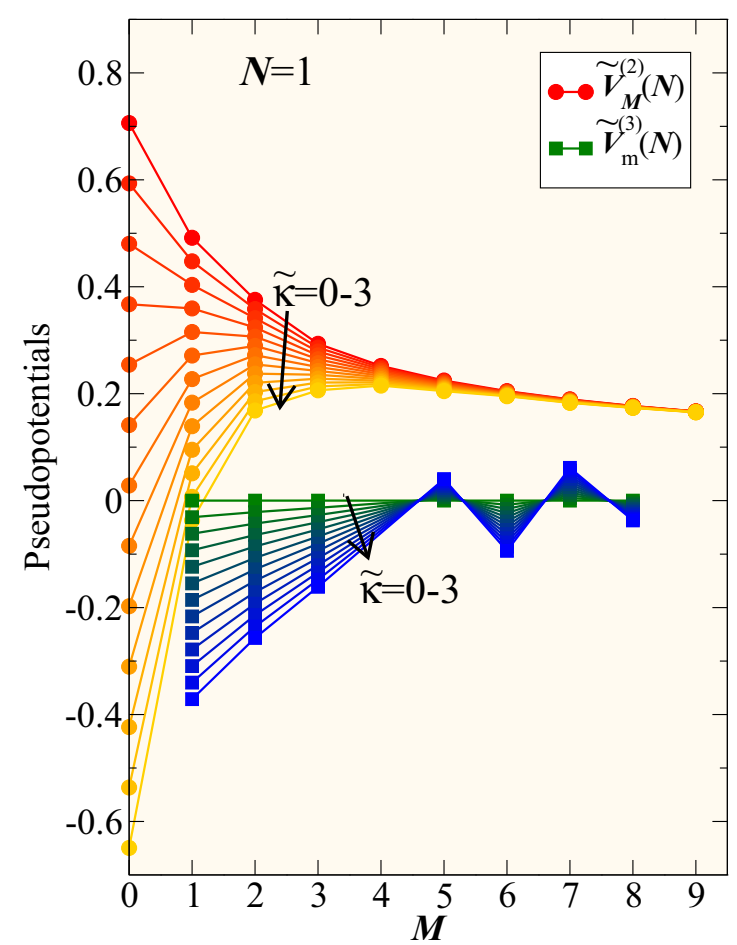

FIG. 2. (Color online) Both the two-body and the three-body pseudopotentials as functions of $M$ for $\tilde{\kappa}=0-3$ for graphene in the $N=1$ Landau level. The $M=6$ three-body term has been corrected. All energies are given in units of $e^{2} / \epsilon \ell_{0}$. This figure replaces the middle panel of Fig. 11 in the original paper. 